\title{
Construction of Wireless Piezoelectric Micro Robots with Arduino Control Module
}

\author{
Chi Hsiang Pan ${ }^{1}$, Hsin-Ying Pan ${ }^{2}$ \\ ${ }^{1}$ Department of Mechanical Engineering / National Chin-Yi University of Technology \\ Taichung, Taiwan, ROC \\ pancs@ncut.edu.tw; \\ ${ }^{2}$ International Bachelor Degree Program on Energy, National Cheng Kung University \\ Taichung, Taiwan, ROC \\ jayshawnpan@gmail.com
}

\begin{abstract}
Based on our previous researches, wireless piezoelectric micro robots integrated with the Arduino control module have been presented. The construction of the robot is included with the frame body, the piezoelectric beams, the miniaturized voltage amplifierpiezo driver, the Bluetooth module, the micro sensor and the Arduino controller (Arduino nano or Rfduion-Simblee). The volume of the robots can be shrunk into a size of several dozen cubic centimeters. In contrast to general mobile micro robots, the actuating mechanism of the robot is piezoelectric bimorph beam(s) and the driving principle is the traveling-wave as drive source to actuate the robot. The robots can move back and forth and rotates counter clockwise or clockwise, respectively. They can be wirelessly controlled by the Bluetooth module of HC06 or Rfduino-Simblee. For further application, the robots can be integrated with micro sensors to interact with the environment parameters. In this paper the HC-SR04 ultrasonic sensor and photo sensitive resistor are used for demonstration.
\end{abstract}

Keywords: Micro robot, Piezoelectric, Traveling wave, Arduino.

\section{Introduction}

Micro robots are attractive to great applications. Many kinds of micro robots have been designed and a wide variety of mechanisms have been investigated for miniaturized robotic locomotion [1]. Different actuators have been adopted for micro robots, such as electromagnetic, pneumatic, electrostatic, shape-memory alloy and piezoelectric actuators. Recently, the piezo-based vibromotor technique with traveling-wave or standing-wave as drive source to actuate the robots has been proposed [2-9]. Over the years Arduino[10] has been the brain of thousands of projects, from everyday objects to complex scientific instruments. Arduino is an open-source electronics platform based on easy-to-use hardware and software. Because it is so flexible and open source, Arduino is the best solution if we are interested in creating interactive objects or environments. Therefore, in this paper, based on our previous researches [8,9], wireless piezoelectric micro robots integrated with the Arduino controller module have been presented. The construction of the robot is included with the frame body, the PZT piezoelectric beams, the miniaturized voltage amplifier-piezo driver, the HC06 Bluetooth module, the micro sensors (HC-SR04 ultrasonic sensor and photo sensitive resistor) and the Arduino controller (Arduino nano or Rfduion-Simblee). The volume of the robot can be shrunk into a size of several dozen cubic centimeters. In contrast to general mobile micro robots, the actuating mechanism of the robot is piezoelectric bimorph beam(s) and the driving principle is the traveling-wave as drive source to actuate the robots. The robots can move back and forth and rotates counterclockwise or clockwise, respectively. They can be wirelessly controlled by the Bluetooth module of HC06 or Rfduino-Simblee. For further application, the robots can be integrated with micro sensors such as the HC-SR04 ultrasonic sensor and the photo sensitive resistor to be interactive with environment.

\section{Construction and Operation Principle of the Piezoelectric Micro Robot}

Figure 1 illustrates the schematic diagram of the construction and operation principle for the mobile piezoelectric micro robot $[8,9]$. The driving principle is the piezo-based traveling-wave method, which applies pulse voltage to excite piezoelectric bimorph beams, and the vibration of the beams causes the frame body to generate surface waves (travelingwave) on the bottom plate of the frame body. When a traveling wave propagates through the surface of the bottom plate, 
each material point on the surface of the bottom plate describes an elliptic motion. If the bottom plate of frame body is placed in contact with the ground, these material points successively enter in contact with the ground and transfer to it, by friction, the mobile micro robot placed on the ground can be moved by the waves. Figure 2 shows four phases of the operations for the robot in motion: the synchronized vibration of the two beams makes the robot move back and forth by adjusting the input pulse signals (frequency) to the piezo-beams, and if only one of beams vibrating, the robot rotates counter clockwise or clockwise, respectively. Figure 3 shows the micro robots: wired or wireless, presented by our previous researches [8,9]. The wireless robot is controlled by Infrared remote control made by ourselves but without integrated with any Arduino controller or Bluetooth module or sensor.
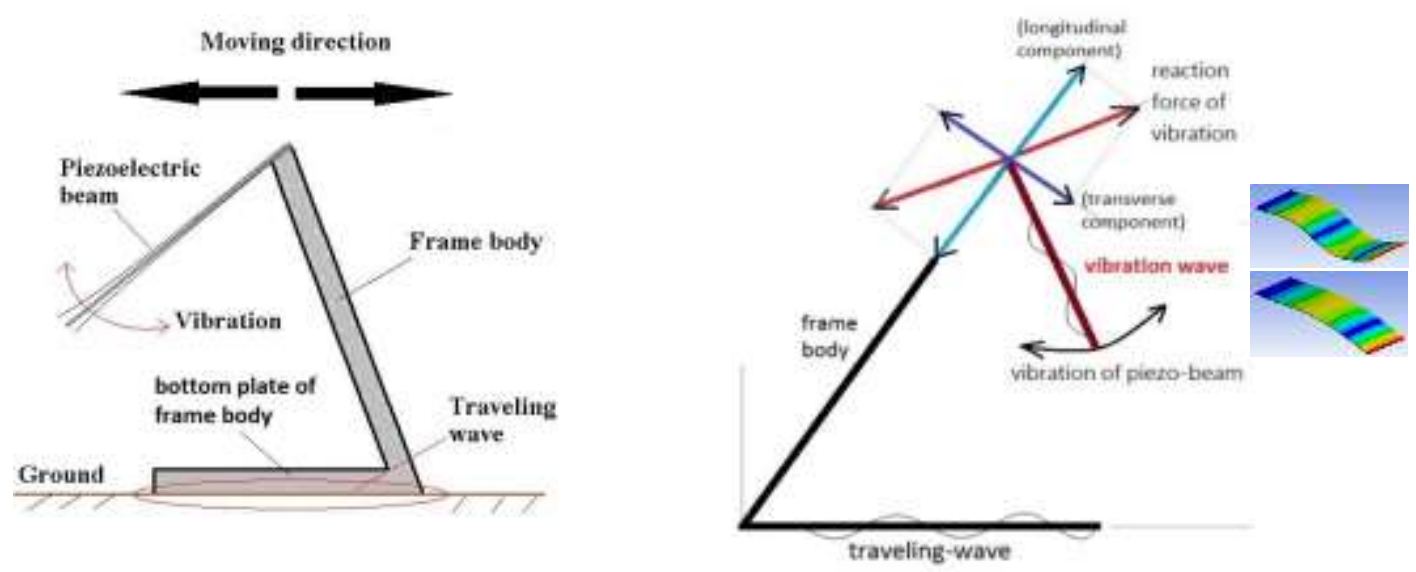

Fig. 1: The schematic diagram of the construction and operation principle for the piezo-based traveling-wave micro robot.

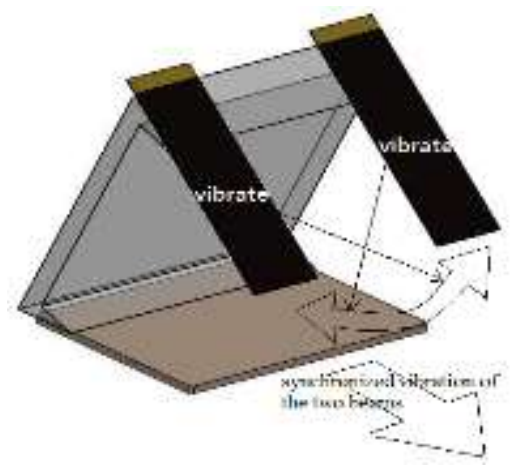

Fig. 2: The phases of the operation for moving the micro robot $[8,9]$.
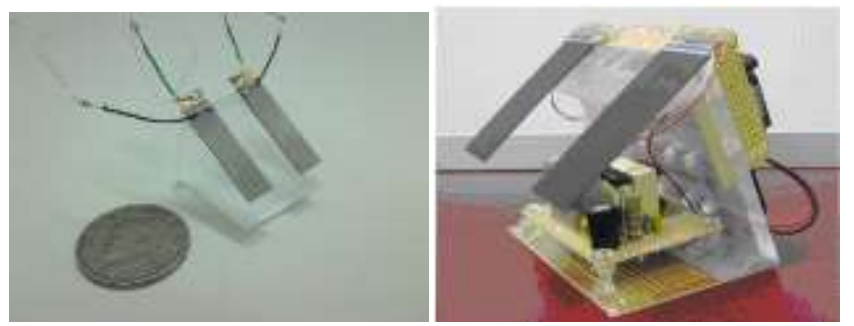

Fig. 3: The wired and wireless piezoelectric micro robots without integrated with Arduino controller module in our previous papers $[8,9]$.

\section{Miniaturized Piezo-Drivers for Driving Piezoelectric Beams}

Figure 4 shows the traditional equipments available in a Lab. for driving the piezoelectric actuators, including the function generator, oscilloscope, power supply, and high voltage amplifier-piezo driver and modulator. However, in 
order to realize the wireless piezoelectric micro robots, the miniaturized piezo-driver must be developed. Two miniaturized piezo-drivers are used in the paper. One is made by ourselves, as shown in Fig. 5, and Figure 5(b) shows the square diagram of the piezo-driver. The size of the micro piezo driver is about $40 \times 50 \mathrm{~mm}$ and weight $35 \mathrm{~g}$. The other miniaturized piezodriver PDu100 shown in Fig. 6 is purchased from the company PiezoDrive [11]. The PDu100 is the industry's smallest and lightest piezoelectric actuator driver. The size of the micro piezo driver is $12 \times 10 \mathrm{~mm}$ and weight $0.5 \mathrm{~g}$.

The piezo-drivers convert a DC input voltage(3 5.5V for PDu100; 5 12V for our made piezo-driver) to an AC output voltage for driving low frequency piezo-beams of the micro robots. Optimum tuning of the voltage and frequency is accomplished by observing the moving conditions of the robots during operation. Output frequency of the piezo-drivers are manually adjusted by turning the resistance on the NE555, as shown in the Fig. 7, and the product description of the NE555 is described as follow:

- Operating voltage: $5 \sim 12 \mathrm{~V}$

- Output voltage: square wave signal amplitude is about equal to the supply voltage

- single-channel signal output, output duty gap of about 50\% square wave module

- adjustable resistance: Adjustable resistance value can control the output frequency

- adjustable frequency range: $2 \sim 25 \mathrm{kHz}$

- Board Size: 25x13mmx13mm (height)

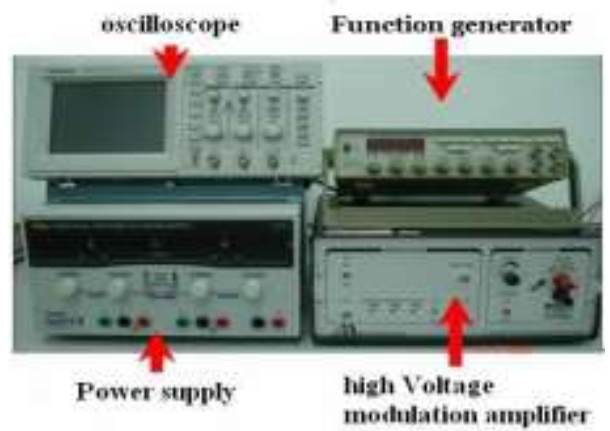

Fig. 4: Equipment in lab. for driving piezoelectric actuators.

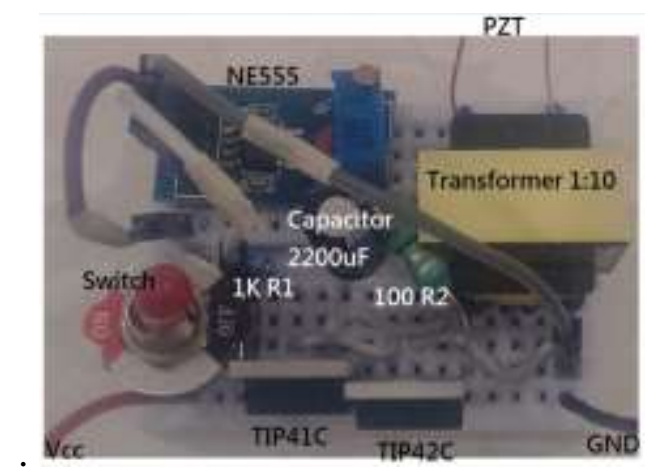

(a)

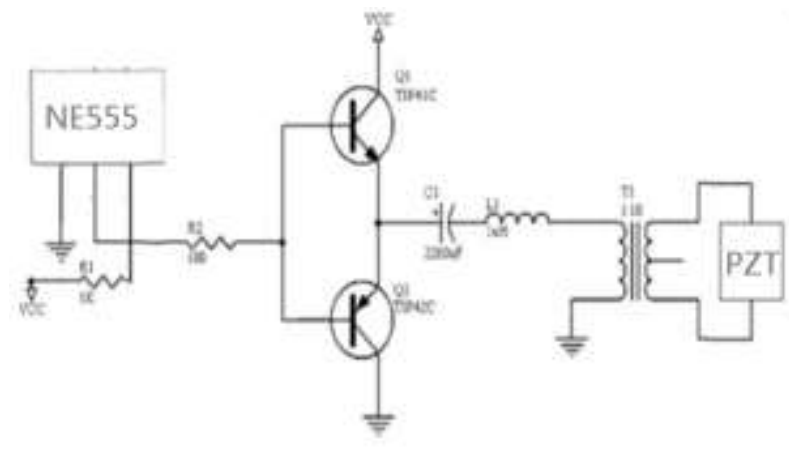

(b)

Fig. 5: The miniaturized piezo-driver made by ourselves. 

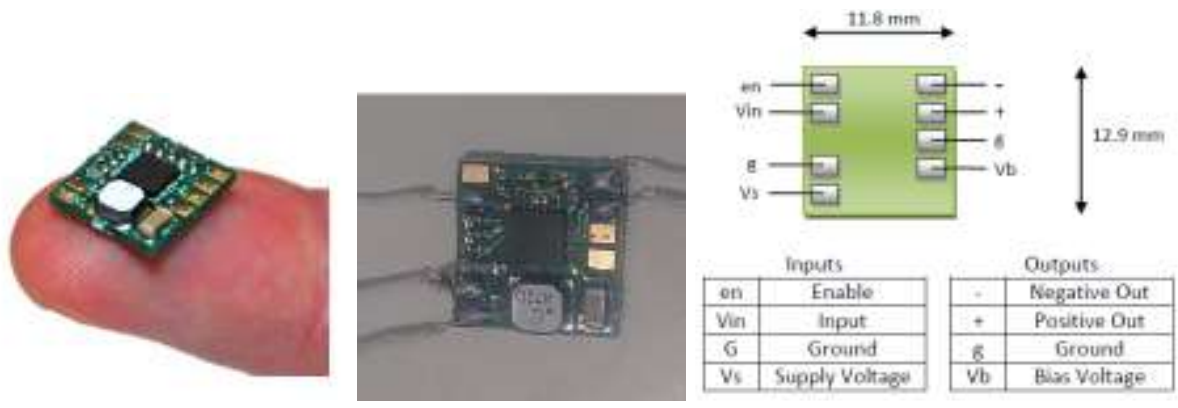

Fig. 6: PDu100 Micro Piezo Driver

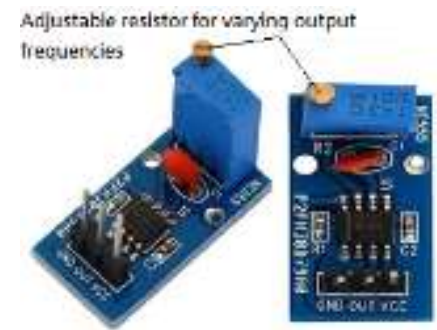

Fig. 7: The square wave signal generator NE555 with Frequency adjustable.

\section{Arduino Controller, HC06 Bluetooth Module and Sensors \\ 4.1. Arduino nano-328}

Figure 8 shows the Arduino nano, which has the advantages of small size, multiple I/O pins, better controllability and compatibility, low power consumption, and can be integrated with Bluetooth or WiFi module, and suitable for mini robots to achieve the desired action. The size is $45 \mathrm{~mm}$ length $\mathrm{x} 18 \mathrm{~mm}$ width. The description of the product is as follow:

- Digital I/O digital input/output terminal 0 13 (digital pin 14 and 6 PWM outputs)

- Analog I/O input analogy 0 7 (8 analog inputs)

- $40 \mathrm{~mA}$ per pin current

- Support USB interface protocol and power supply

- Support ISP download function

- Support single chip TX/RX terminal

- Support AREF terminal

- Supports 6 PWM terminals (Pin11, 10, 9, 6, 5, 3)

- Input voltage: external power supply 6V 12V DC, no external power supply needed when connected to USB

- Output voltage: 5V DC \& 3.3V DC

\subsection{Rfduino-Simblee}
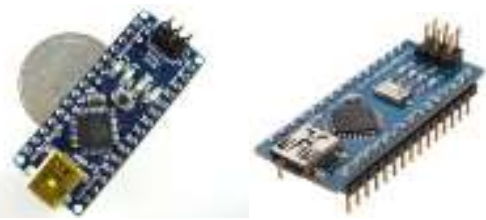

Fig. 8: Arduino nano-328.

Figure 9 shows the Simblee, which is designed and developed by Rfduino, a company focused on creating wireless devices that can be programmed with the Arduino IDE. The Simblee is a BLE module that contains both a BLE radio and a 
32-bit ARM Cortex M0 processor with $128 \mathrm{~KB}$ of flash memory, 24KB of RAM, and operates at 16 MHZ. The RFduinoSimblee is burned by the RFD22121 USB port(Fig, 9(b)). It can be removed after burning. Provide 3.3V regulator supply to Rfduino. The description of the product as follow:

- Operating voltage: $3 \mathrm{~V}$ (maximum $3.6 \mathrm{~V}$, low $1.8 \mathrm{~V}$ )

- Transmit/receive current: 8-16mA (<3uA ULP)

- Build iPhone and Android apps without Xcode or Android SDK

- Built-in AES encryption engine

- $-93 \mathrm{dBm}$ receiver sensitivity

- $-55 \mathrm{dBm}$ to $+4 \mathrm{dBm}$ TX power

- 7 GPIO pins (6 10-bit analog inputs)

- PWM, UART, SPI, I2C functions on most GPIO pins

- UART programming or over-the-air programming via USB

- CE, ETSI, IC, TELEC and FCC approval

- $28.96 \mathrm{~mm}$ x $22.86 \mathrm{~mm}$ x $18.95 \mathrm{~mm}$
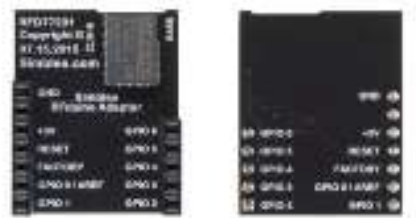

(a)

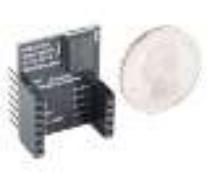

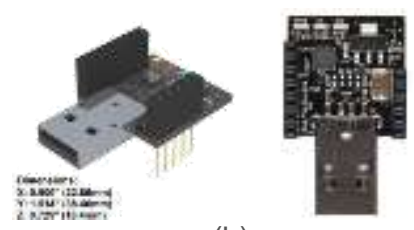

(b)

Fig. 9: (a) RFduino - Simblee DIP (RFD77201 Simblee 7-pin DIP); (b) RFD22121 USB Programming Shield.

\subsection{HC06 Bluetooth Module}

The HC-06 Bluetooth module (Fig. 10) is a slave Bluetooth module designed for wireless serial communication. It is a slave module meaning that it can receive serial data when serial data is sent out from a master Bluetooth device (device able to send serial data through the air: smart phones, PC). When the module receives wireless data, it is sent out through the serial interface exactly at it is received. No source code specific to the Bluetooth module is needed at all in the Arduino chip. An app on the phone is used to send out inputs to the module which receives and then transfers this to the Arduino. The Arduino and actuators in turn responds accordingly, as specified in the source code. When the module is not in a paired state, the LED on the module blinks rapidly whereas when paired with the app on the phone, the LED on the module is a steady red.

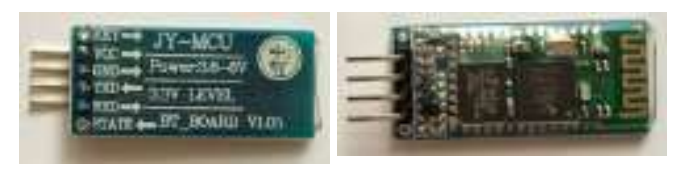

Fig. 10: HC-06 bluetooth module.

\subsection{Sensor- Photosensitive Resistor}

Photosensitive resistors(Fig. 11) are also called light pipes. The commonly used materials are cadmium sulfide, and there are also materials such as selenium, aluminum sulfide, lead sulfide and barium sulfide. These materials have the property that their resistance rapidly decreases under the illumination of light of a specific wavelength. In this paper, Keyes K853518 Photosensitive Sensor for Arduino is used. Power should be 3V - 5.5V DC power supply. The output is analog. Dimensions: 1.14 in $x 0.87$ in $x 0.39$ in

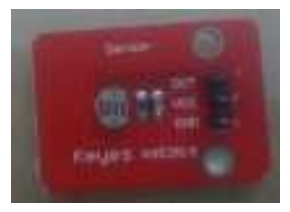

Fig. 11: Photosensitive resistors. 


\subsection{Sensor-Ultrasonic Sensor HC-SR04}

The HC-SR04 ultrasonic sensor (Fig. 12) uses sonar to determine distance to an object like bats do. It offers excellent non-contact range detection with high accuracy and stable readings in an easy-to-use package. From $2 \mathrm{~cm}$ to $400 \mathrm{~cm}$ or 1 " to 13 feet, Its operation is not affected by sunlight or black material like sharp rangefinders are (although acoustically soft materials like cloth can be difficult to detect). It comes complete with ultrasonic transmitter and receiver module. The description of the product as follow:

- $\quad$ Power Supply :+5V DC -- Quiescent Current : $<2 \mathrm{~mA} \quad$-- Working Current: $15 \mathrm{~mA} \quad$-- Effectual Angle: $<15^{\circ}$

- Ranging Distance : $2 \mathrm{~cm}-400 \mathrm{~cm} / 1^{\prime \prime}-13 \mathrm{ft} \quad$ Resolution : $0.3 \mathrm{~cm} \quad$ Measuring Angle: 30 degree

- Trigger Input Pulse width: $10 \mathrm{uS} \quad$ Dimension: $45 \mathrm{~mm}$ x $20 \mathrm{~mm}$ x $15 \mathrm{~mm}$

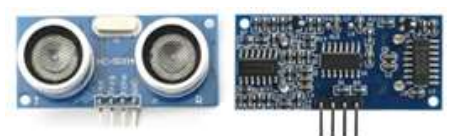

Fig. 12: The HC-SR04 ultrasonic sensor.

\section{Assembly and Test of the Micro Robots}

In this section, assembly and study on the performances of the micro robots experimentally is presented. The influences of driving voltage and driving frequency on the speed of the robots are investigated. The speed is measured by timer and ruler. According to previous researches $[8,9]$, the material, the geometry of the frame body and the gap distance between two piezoelectric beams are significant to affect the performance of the robot. In addition, different ground surfaces (material, roughness or hardness) affect the speed of the robot. For demonstrations, the frame bodies of the micro robots are made of 3D printing PLA materials. The geometry of the frame body and the gap distance between two piezoelectric beams are shown in Fig. 13. The volumes are about $50 \sim 100 \mathrm{~cm}^{3}$ and the weights are tens to hundreds of grams (including all the components of the robot).

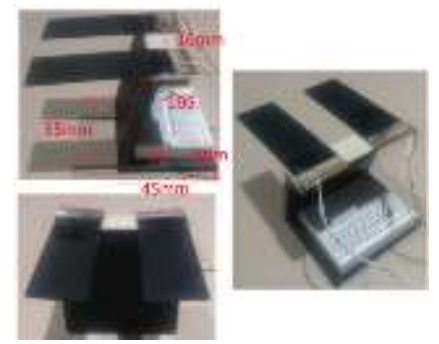

Fig. 13: The geometry of the frame body and the gap distance between two piezoelectric beams.

Figure 14 shows the one piezo-beam micro robot integrated with, Arduino nano, self-made miniaturized Piezo-driver, HC06 Bluetooth and HC-SR04 ultrasonic sensor, and Figure 15 shows the schematic (block) diagram of Arduino nano integrated with mechatronic components. The Arduino nano is the central processing unit of the micro robot. A total of 9 pins were used to connect the battery (Vin pin), relay(D12 pin), sensors(D8,9) and Bluetooth(D2,3). In this case, two batteries needed to supply the powers for the Arduino nano and the piezo-driver (micro robot). Since Arduino nano only outputs $3.3 \sim 5 \mathrm{~V}(3 \mathrm{~V} 3$ pin, $+5 \mathrm{~V}$ pin), extra battery to provide larger voltage $(>5 \mathrm{~V})$ for the piezo-driver is used. The software code for this robot has been divided into two operation modes: autonomous mode and remote control mode. Two modes can be switched by using the Bluetooth app. The functionalities were set and adjusted using Arduino IDE. In remote control mode, the robot is controlled by using the Bluetooth app of the smart phone to send commands to the Arduino. In the autonomous mode, the ultrasonic sensor will take over robot throughout its journey. Figure 16 shows the micro piezoelectric robots (one or two piezo-beams) integrated with Rduino-Simblee, PDu100 Micro Piezo Driver and Photosensitive resistor. In this case only one operation modes: remote control mode. Remote control is operated through the Simblee Bluetooth by using i-phone Simblee app only. The Photosensitive resistor will take over robot throughout its journey to stop or to move according the illumination. 

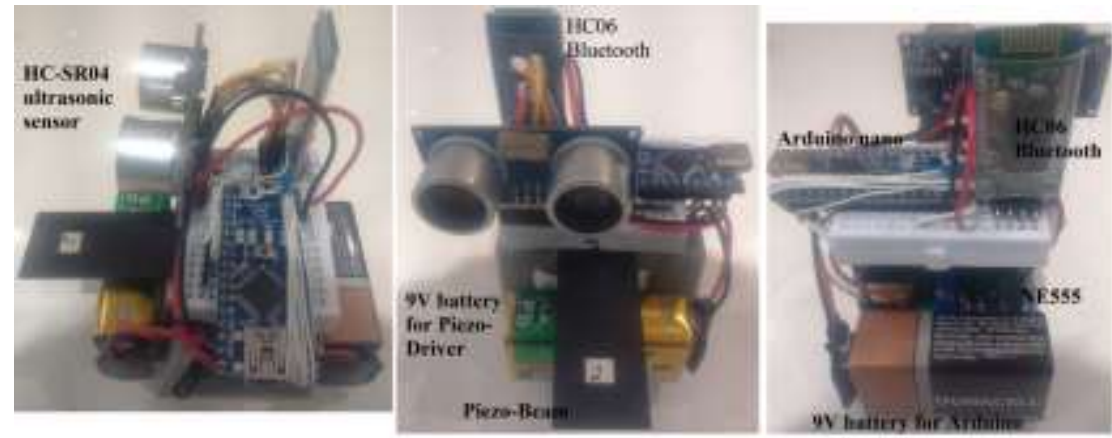

Fig. 14: Micro piezoelectric robot integrated with Arduino nano, self-made miniaturized Piezo-driver, HC06 Bluetooth and HC-SR04 ultrasonic sensor.

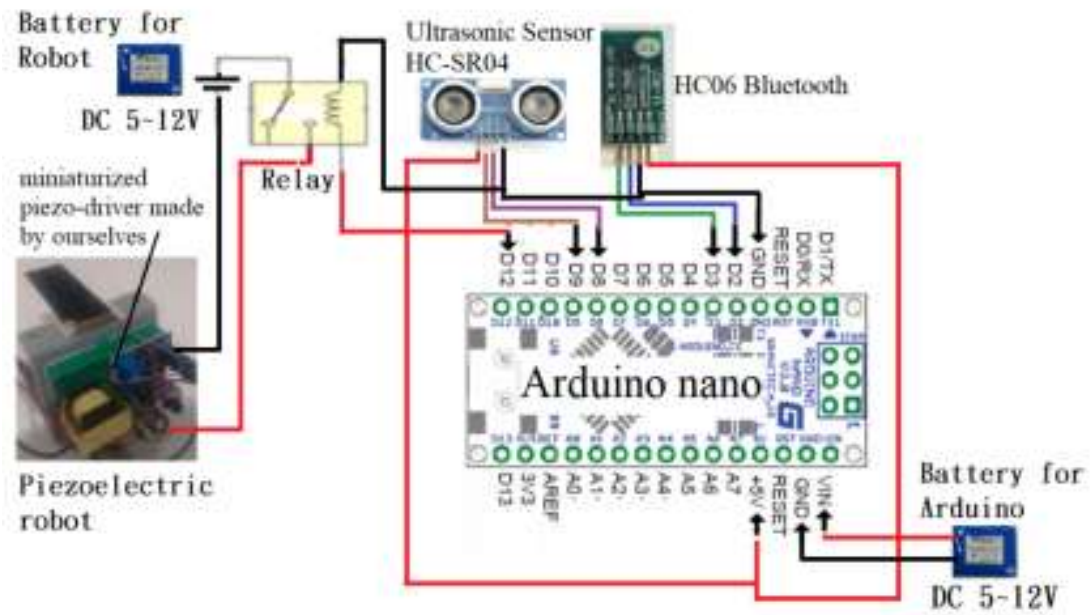

Fig. 15: Schematic (block) diagram of Arduino nano integrated with mechatronic components.

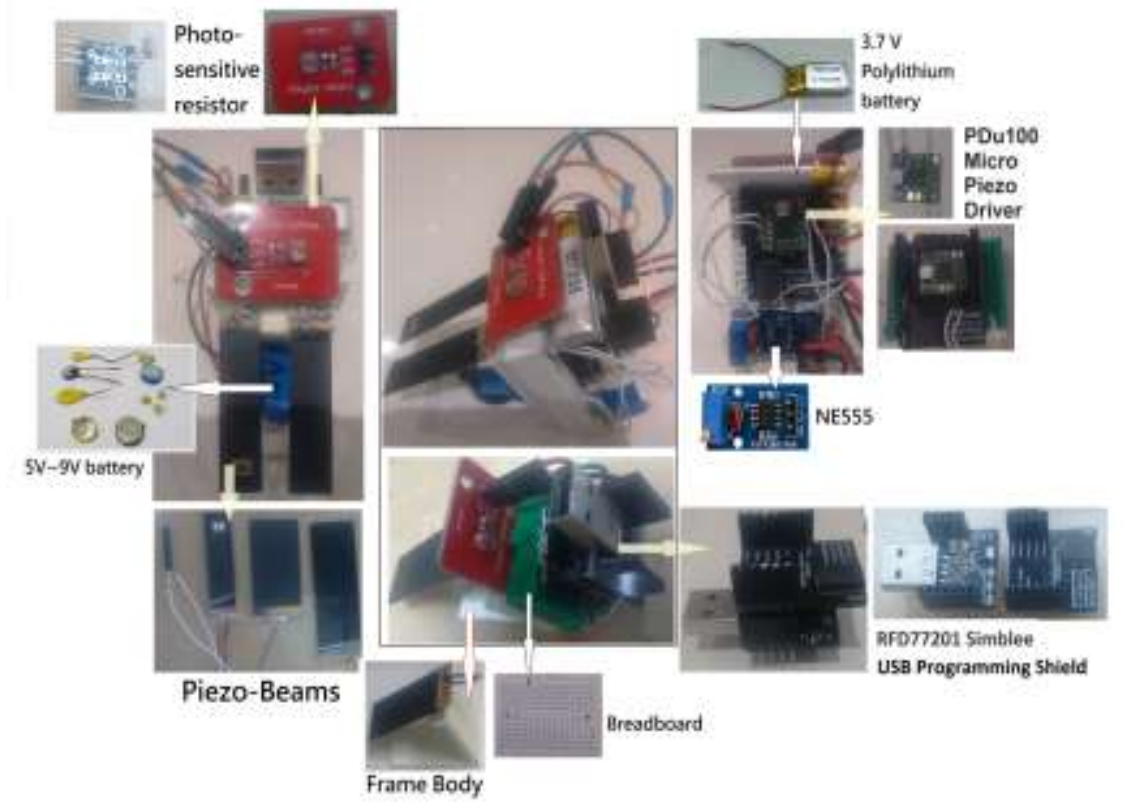

Fig. 16: Micro piezoelectric robot integrated with Rduino-Simblee, PDu100 Micro Piezo Driver and Photosensitive resistor 
Experimental tests of the micro robots with different driving frequencies are performed. Tuning of the frequency is accomplished by observing the moving conditions of the robots during operation. Output frequency of the piezo-driver is manually adjusted by turning the resistance on the NE555. The robots are operated on a smooth glass substrate. According to the results, it is found that the driving frequency affect the speed (magnitude and direction) of the robots sensitively and significantly, even change the moving direction of the robot. This is due to the generated travelling wave changes wave propagation direction according to the different driving frequencies. Improper driving frequencies may make the speed slow down sharply, even zero speed, due to no generation of a traveling wave on the bottom plate of the frame body. Therefore, modulating the frequency for optimal driving frequency is very important to give the better travelling wave performance and to obtain the higher speed. By the way, if we can control the driving frequencies properly, we can drive the robot to move back and forth and to rotate counter clockwise or clockwise, respectively. According to experimental results, it is found that a larger voltage will produce higher speed for the robot.

\section{Conclusion}

We have successfully integrated mobile piezoelectric micro robots with the Arduino controlled module, which are different from our previous papers $[8,9]$. Based on the experimental characterization, the performance of the micro robot was conducted on the key parameters, including the drive frequency and drive voltages. It is found that the driving frequency affect the speed (magnitude and direction) of the robots sensitively and significantly. For demonstration, two types of the micro robots with a size of several dozen cubic centimeters are developed:(1) one piezo-beam micro robot integrated with, Arduino nano, self-made miniaturized Piezo-driver, HC06 Bluetooth and HC-SR04 ultrasonic sensor; (2) The micro robots (one or two piezo-beams) integrated with Rduino-Simblee, PDu100 Micro Piezo Driver and Photosensitive resistor. Based on the study, more smart functions can be constructed with various sensors, wireless modules and programing in the future.

\section{References}

[1] S. Fatikow and U. Rembold, Microsystem Technology and Microrobotics. Springer, 1997.

[2] H. Hariri, Y. Bernard, A. "Razek, Modeling and Experimental Study of a Two Modes Excitation Travelling Wave Piezoelectric Miniature Robot," ACTUATOR 2012, 13th International Conference on New Actuators, Bremen, Germany, 18-20 June 2012

[3] R. Bansevicius, A. Drukteiniene, G. Kulvietis and I. Tumasoniene, "Design of a Mobile Microrobot Based on Standing and Travelling Waves," Int J Adv Robotic Sy, vol. 10, pp. 219, 2013.

[4] H. Hariri, Y. Bernard, A. Razek, "Traveling wave piezoelectric beam robot," Journal of Smart Mat. \& struct., vol. 23, no. 2, 2014.

[5] Y. Roh, J. Kwon, "Development of a new standing wave type ultrasonic linear motor," Sensors and actuators A, vol. 112, pp. 196-202, 2004.

[6] H. H. Hariri, G. S. Soh, S. H. Foong, K. L. Wood, K. Otto, "Miniature Piezoelectric Mobile Robot driven by StandingWave," in the 14th IFToMM World Congress, Taipei, Taiwan, October 25-30, 2015

[7] E. B. Diann, J. Blechschmidt, "Design and Static Modeling of a Semicircular Polymeric Piezoelectric Microactuator," Journal of Microelectromechanical Systems, vol. 1, no. 3, pp. 106-115, 1992.

[8] C. H. Pan, Sz sheng tzou, Ruei yang shiu, "A Novel Wireless and Mobile Piezoelectric Micro Robot," in Proceedings of the 2010 IEEE International Conference on Mechatronics and Automation, Aug. 4-7, Xidian, China, pp. 1158-1163.

[9] C. H. pan (2016, Jul). "Novel Mobile Piezoelectric Micro Robots Driven by Traveling Wave," in the First International Conference on Manipulation, Automation and Robotics at Small Scales, Paris, France, 2016.

[10] Arduino. [Online]. Available: https://www.arduino.cc/

[11] PiezoDrive. [Online]. Available: https://www.piezodrive.com/modules/pdu100-micro-piezo-driver/ 